\title{
DIFFUSION DU COTON GÉNÉTIQUEMENT MODIFIÉ EN AFRIQUE DU SUD : DES LEÇONS POUR L'AFRIQUE ZONE FRANC
}

\author{
Jean-Luc HOFS *, Michel FOK *, Marnus GOUSE **, \\ JOhann KIRSTEN**
}

\begin{abstract}
Si l'utilisation du coton génétiquement modifié (CGM) en Afrique du Sud, depuis 1996-1997, est le plus souvent présentée comme un succès d'adoption d'Ogm par les petits paysans de pays en développement, c'est une présentation qui occulte le fait que la production cotonnière est d'abord celle des fermiers blancs. Le succès du CGM dans ce pays est aussi tout relatif car la production continue à décliner et la part des petits paysans noirs reste toujours aussi faible.

Le cas de l'Afrique du Sud montre que la seule introduction d'une technologie nouvelle ne suffit pas pour assurer la progression de la production à l'échelle du pays. Des facteurs d'ordre institutionnel interviennent, mais la focalisation exclusive sur le CGM les occulte.

Nos travaux fondés sur une enquête récente mettent en évidence une mauvaise rentabilité dans une année défavorable sur le plan climatique et institutionnel. Dans des conditions plus favorables, la rentabilité est meilleure, mais avec un risque financier accru.

Rares sont les pays de l'Afrique Zone Franc présentant aujourd'bui une stabilité institutionnelle favorable à l'introduction du CGM. Celle-ci nécessite préparation notamment pour négocier des conditions économiques favorables de transfert de la biotechnologie et pour une réorganisation de la distribution de semences préservant les intérêts des paysans. Les difficultés associées à cette préparation mettent en garde contre une adoption précipitée.
\end{abstract}

Beaucoup d'études récentes démontrent l'efficacité et la rentabilité des variétés génétiquement modifiées en agriculture (EDEN, SLINSKY, LARSON, ROBERTS et

* CIRAD-UPR 10, TA 72/09.

** Department of Agricultural Economics, University of Pretoria, South Africa. 
LENTZ, 1998 ; MARRA, PARDEY et ALSTON, 2003). L'accent est particulièrement mis sur le coton génétiquement modifié (CGM), notamment les variétés résistantes aux chenilles de capsules du cotonnier incorporant un gène $\mathrm{Bt}$ (coton $\mathrm{Bt}$ ). L'intérêt de l'utilisation de variétés génétiquement modifiées dans les pays en développement (PVD) fait cependant encore l'objet de débats. Les opposants aux OGM doutent de leur compatibilité avec les besoins des petits producteurs des PVD (MAZOYER, 2000 ; MYERS, 1999), ils s'interrogent sur leur efficacité et leur rentabilité (DE GrASSI, 2003) en raison de la cherté des semences que devrait renforcer l'instauration d'une situation monopolistique dans la fourniture de ces dernières (PSCHORNSTRAUSS, 2004).

Les PVD, notamment en Afrique, ne veulent pas se fermer à l'utilisation du CGM. L'Afrique du Sud l'a déjà adopté dès 1997-1998 et le Burkina Faso a initié les expérimentations depuis 2002 (MANÉ, 2005), alors que les paysans du Mali viennent de faire connaître leur opposition à l'introduction du CGM (SAMPHIER et CARLILE, 2006). Les expériences positives dans des pays présentés comme des PVD (la Chine, l'Afrique du Sud et plus récemment l'Inde) ont contribué à alimenter l'idée que le CGM serait adapté aux PVD en général et mériterait le soutien (HOAG, 2003) que les Américains ont déjà décidé d'apporter (MOORJANI, 2005).

Les résultats rapportés positifs de l'utilisation du CGM concernent le gain de rentabilité (découlant d'une réduction du nombre de traitements insecticides et d'une augmentation du rendement) et la diminution de la quantité et de la pénibilité du travail. Ces résultats sont cependant obtenus sur une durée assez courte. Leur extrapolation à d'autres pays est aussi difficile car ils sont souvent présentés sans tenir compte des contextes locaux de production et d'organisation de la filière cotonnière, facteurs dont on connaît l'importance dans la production par les petits paysans.

L'objet de cet article est de restituer l'expérience sud-africaine de l'utilisation du CGM par les petits paysans, en exploitant en particulier les résultats d'une enquête conduite lors de la campagne 2002-2003. Nous voulons nuancer les résultats rapportés par les travaux antérieurs en soulignant l'instabilité de la rentabilité et le changement significatif de la structure des coûts de production. Ce dernier changement a pour effet d'accentuer le risque financier associé à l'utilisation du CGM. Tout en confirmant certains aspects positifs de la commercialisation du CGM en Afrique du Sud, notre but est d'éclairer aussi sur certains effets négatifs insuffisamment pris en compte dans la littérature. Il en découle une série d'enseignements utiles aux pays de l'Afrique Zone Franc, pressés aujourd'hui de s'engager dans la voie du CGM.

Dans cet article, la première partie informe sur les caractéristiques de la production cotonnière en Afrique du Sud. La deuxième partie fait la synthèse des résultats de travaux antérieurs sur les impacts de l'utilisation du CGM en Afrique du Sud et dans d'autres pays. Dans la troisième partie, nous présenterons les résultats de notre enquête pour décrire les particularités des structures de production paysannes et les résultats économiques de l'utilisation du CGM, dans une année cependant très défavorable tant sur le plan climatique qu'institutionnel. Avant de conclure, la quatrième partie est consacrée à expliciter les divers enseignements utiles à l'Afrique Zone Franc. 


\section{I - UNE PRODUCTION DÉCLINANTE, TENDANCE PERSISTANTE}

En dépit de la libéralisation de la filière en 1994, la production cotonnière baisse en Afrique du Sud et la contribution des petits paysans reste très faible.

\section{1 - Une Contribution minime du petit paysannat à la production cotonnière}

La production cotonnière provient à la fois des fermiers blancs et des paysans noirs. Les premiers cultivent sur une surface relativement grande (le plus souvent 50-60 ha) avec une irrigation assez fréquente, alors que les deuxièmes cultivent le plus souvent quelques hectares et sans irrigation. La production des petits paysans noirs est aujourd'hui localisée essentiellement dans le Kwazulu Natal, à la frontière du Swaziland et du Mozambique, sur le Plateau des Makhatini Flats. Les précipitations annuelles y sont de $550 \mathrm{~mm}$, réparties entre septembre et début mai. C'est dans cette zone que nous avons réalisé notre enquête, comme les travaux antérieurs d'autres équipes, mais lors de la campagne 2002-2003 marquée par des pluies tardives et leur arrêt précoce.

Aujourd'hui, la production cotonnière en Afrique du Sud est d'environ 20000 tonnes de fibre, très loin du record de 1988 (figure 1) suivant une baisse brutale à partir de 1989. La libéralisation de la filière (cf. infra), mise en ouvre lors de la campagne 1994-1995, n'a pas induit une reprise durable des emblavements cotonniers. Avec une industrie textile qui a pourtant du mal à se maintenir, les importations sont actuellement proches du double de la production nationale.

La production cotonnière par les petits paysans noirs était faible avant la libéralisation ; elle a diminué après. En termes relatifs, la contribution des petits paysans à la production nationale reste très faible, la remontée de cette contribution les deux dernières années est trompeuse car elle est liée à la baisse globale de la production nationale (tableau 1, p. 816). Depuis la libéralisation de la filière, le nombre de producteurs de coton parmi les petits paysans des Makhatini Flats a fluctué autour de 3 000, jusqu'à la campagne 2001-2002, mais pour une superficie qui a chuté de 10000 ha à environ 3000 ha. Lors de la campagne 2002-2003, l'incertitude sur l'octroi du crédit-intrant et le retard avec lequel il a été finalement fourni ont induit un effondrement du nombre de producteurs de coton. Jusqu'en 2002-2003, le rendement en fibre fluctue autour de $200 \mathrm{~kg} / \mathrm{ha}$ ou autour de $540 \mathrm{~kg} / \mathrm{ha}$ pour le coton-graine, soit au mieux la moitié de ce qui est observé en Afrique francophone (environ $1100 \mathrm{~kg} / \mathrm{ha}$ ). Depuis les deux dernières campagnes, le rendement moyen a fortement augmenté en raison d'une part plus importante de la production en irrigué.

Le coton en Afrique du Sud n'a donc pas la qualité de success story. Les petits paysans sont peu impliqués, la production décline, les besoins nationaux ne sont pas couverts. Le seul apport du recours au CGM concerne le rendement moyen au niveau national. Ce dernier est passé de moins de $400 \mathrm{~kg} / \mathrm{ha}$ de fibre à une moyenne de $500 \mathrm{~kg} / \mathrm{ha}$ : c'est un gain de $25 \%$ que l'on peut attribuer à la fois à l'adoption du CGM et à la plus grande contribution de la production irriguée et des grands fermiers (dont le rendement moyen est plus élevé). 
Figure 1 : Évolution fluctuante et tendance baissière de la production cotonnière en Afrique du Sud

(Fluctuating evolution and downward trend of cotton production)

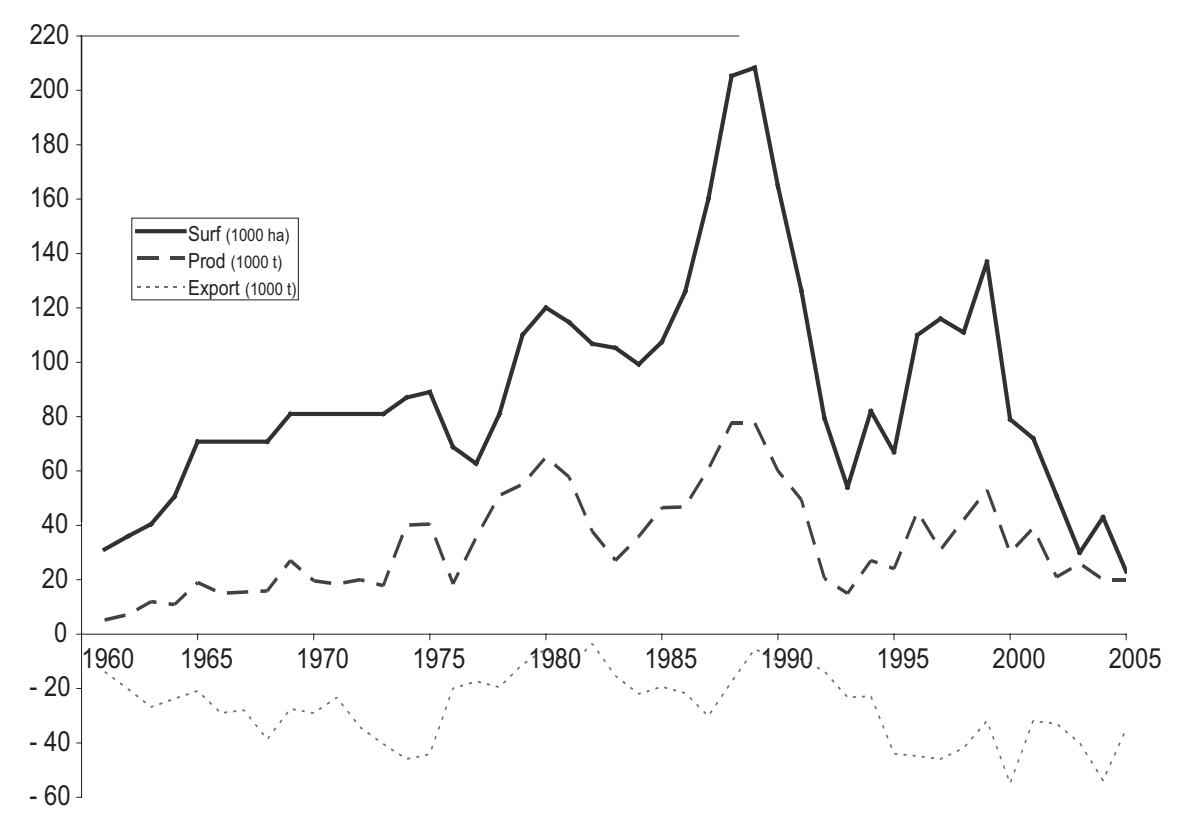

Source : Cotton South Africa.

\section{2 - Un monopole privé sans contrôle après la libéralisation}

Les conditions de production par les petits paysans ne se sont pas améliorées depuis la libéralisation, cette une évolution s'est faite en plusieurs étapes.

\section{a - D’une fillière adimilnistrée à la libéralisation}

Le coton a une longue histoire en Afrique du Sud, mais c'est seulement en 1974 qu'une politique cotonnière fut élaborée avec la création de l'Office du coton (Cotton Board) (MACRAE, 1995). En-dehors des rôles importants dans la tenue des statistiques et du classement tant du coton-graine que du coton fibre, l'Office du coton fixait pour les usines les quotas de coton-graine à égrener ainsi que les quotas de fibre à répartir entre les usines textiles. Il administrait aussi le prix d'achat du coton-graine dans le cadre du Cotton Marketing Committee.

La fixation du prix était réalisée en impliquant les associations de producteurs et celles de l'industrie textile. En dépit de la volonté de garantir un prix d'achat supérieur au prix mondial, le prix décidé paraissait toujours insuffisant au regard des coûts de production. À la baisse de la production qui résulta de cette appréciation négative du prix, la perception d'une certaine inefficacité des services agricoles poussa le gouvernement à libéraliser la filière. Cette réforme intervint 
lors de la campagne 1994-1995, avec notamment l'abolition du Cotton Board et l'abandon du prix administré.

\section{b - Une entente entre opérateurs privés et la défaillance du crédit des intrants}

Dans les années 1980, deux sociétés opéraient dans les Makhatini Flats pour fournir crédits et intrants aux paysans et pour leur acheter la production cotonnière. Comme en Afrique francophone, le crédit était remboursé par un prélèvement à la source lors de la commercialisation du coton-graine. L'esprit de coopération entre les deux sociétés les poussa à se fondre dans une société conjointe, appelée Vunisa, en 1989. C'est cette société qui devint la seule à intervenir après la libéralisation, en fournissant le crédit-intrant en partenariat avec la Land Bank. Ce système se révéla cependant insuffisant pour développer réellement la production.

\section{c - D’un monopole privé à un autre}

Le désordre institutionnel est survenu lors de la campagne 2001-2002, lorsqu'une nouvelle société, Makhatini Cotton Gin (Pty) Ltd (MCG), construisit une nouvelle usine d'égrenage juste à côté du dépôt d'achat de la Vunisa dont l'usine se situait à plus de $100 \mathrm{~km}$. La MCG parvint à capter la production des paysans qui avaient emprunté à la Vunisa. Pour éviter les pertes, en 2002-2003, cette dernière décida, avec sa banque partenaire, de limiter l'octroi du crédit au faible nombre de paysans jugés fiables, d'où l'effondrement du nombre de producteurs de coton durant cette campagne. Incapable de suivre l'augmentation du prix d'achat que la MCG engagea après, la Vunisa cessa finalement de fonctionner en 2003-2004 pour laisser la place à la seule MCG.

\section{d - Un processus de forclusion associé à la production irriguée}

En même temps que la MCG établissait son usine d'égrenage, elle réussit à acquérir les droits de propriété d'une grande surface en vue de procéder à une production en régie, sous irrigation. Cette acquisition a été réalisée auprès des autorités coutumières suivant des termes qu'il serait difficile de percer à jour. Elle s'est traduite de fait par la forclusion du droit d'usage de ces terres pour les paysans qui en jouissaient jusqu'alors selon les règles coutumières. Ce processus a permis à la MCG de cultiver 500 ha sous irrigation dès la campagne 2002-2003, puis 1300 ha et 3000 ha les deux campagnes suivantes.

Parallèlement, la MCG mit en ouvre un système contractuel de longue durée pour impliquer les petits paysans dans la production irriguée. Les paysans jugés dignes d'en faire partie, en raison de leur localisation proche de l'usine et de leurs aptitudes techniques, évaluées après une formation, ont signé un contrat de neuf ans qui stipule ceci :

- ils doivent fournir chacun dix hectares ;

- ils reçoivent un dédommagement pour les terres fournies (2000 Rand/ha ou 267 US\$) ; 
- les terres sont équipées en système d'irrigation dont le coût devra être remboursé ;

- un prêt de 20000 Rand leur est consenti la première année, pour produire ;

- ils respectent un plan de remboursement des équipements et des crédits leur laissant $25 \%$ du revenu net, après remboursement de l'annuité des équipements et des intrants pris pour chaque campagne ;

- ils deviennent propriétaires des terres au bout des neuf années.

- ils s'engagent à continuer à traiter exclusivement avec la MCG même après la période de neuf ans (pour acheter les intrants et pour vendre le coton produit).

Aujourd'hui, environ 50 paysans sont impliqués dans ce système. Les paysans non intégrés ne bénéficient plus d'aucun soutien pour produire. Faute d'alternative, ils continuent tout de même à produire du coton, mais en payant les intrants au comptant et sans bénéficier du moindre conseil technique. Ils vendent individuellement leur production à la MCG, qui en fixe le prix.

\section{II - LA RENTABILITÉ DU CGM EN AFRIQUE DU SUD : LES ENSEIGNEMENTS}

\section{1 - Travaux d'évaluation des impacts}

La commercialisation du CGM, plus précisément du coton Bt, a débuté lors de la campagne 1997-1998. Les études, initiées peu après, visaient à cerner rentabilité et réponse aux contraintes, notamment au niveau des petits paysans.

Les résultats de ces travaux ont contribué à faire percevoir l'expérience sudafricaine comme un succès d'utilisation du coton Bt par les petits paysans (MORSE, BennetT et IsmaëL, 2004 ; IsmaëL, Beyers, Thirtle et PIEsse, 2002), même si la portée du triomphe technologique a été récemment nuancée par l'écueil des contraintes institutionnelles (GOUSE, KIRSTEN, SHANKAR et ThIRTLE, 2005).

Depuis la campagne 2001-2002, le CIRAD s'est associé à l'Université de Pretoria pour une évaluation plus technique de l'utilisation du coton Bt. Il s'agissait de cerner l'expression et l'efficacité du gène Bt contre les ravageurs cibles, d'appréhender le phénomène de flux de gènes et le changement éventuel du complexe parasitaire du cotonnier quelques années après la culture du coton Bt. En complément, une enquête a été conduite pour comprendre les modes de culture du cotonnier en petit paysannat, les résultats sont rapportés dans la troisième partie.

\section{2 - Gain de rentabilité et faible niveau de rendement}

Les impacts économiques de l'adoption du CGM par le petit paysannat en Afrique du Sud sont appréciés en référence à d'autres pays, comme la Chine (XU, 
You, Wang et Yang, 2004 ; Pray, Huang, Hu et Rozelle, 2002), l'Inde (BennetT, ISMAËL, KAMBHAMPATI et MORSE, 2004 ; ORPHAL, 2005), l'Argentine (QAIM et DE Janvry, 2003), le Mexique (Traxler et Godoy-Avila, 2004 ; FaO, 2004), ou les États-Unis (FITT, 2003 ; KERBY, 2001), à partir de documents grâce auxquels on a pu recalculer les indicateurs que nous avons retenus. Nous prenons ainsi en compte les pays où le coton Bt est utilisé à grande échelle, mais suivant des modes de production très différents et ne relevant pas forcément du petit paysannat.

\section{a - Un gain très élevé en partant d'un niveau de rendement très bas}

Comme évoqué plus haut, en Afrique du Sud le rendement moyen en petit paysannat est très bas et se situe autour de $540 \mathrm{~kg} / \mathrm{ha}$ de coton-graine depuis la libéralisation de la filière en 1994 (tableau 2, p. 817). Le gain relatif du rendement induit par l'utilisation du coton Bt est ainsi le plus élevé de tous les pays utilisant le coton Bt (tableau 2 et tableau 4, p. 818). Dans la plupart des pays, les gains de rendement se situent autour de $10 \%$ (avec un résultat discordant et étonnant pour la Chine). Clairement, l'utilisation du coton Bt en Australie, championne du monde en termes de rendements pour le coton, n'a pas induit de gain de rendement. C'est aussi le cas dans une province de la Vallée du Fleuve Yangse en Chine, où la pression parasitaire est moindre (XU, YOU, WANG et YANG, 2004), contrairement aux observations dans la Vallée du Fleuve Jaune (PrAY, HUANG, Hu et ROZELLE, 2002).

La même observation vaut aussi pour le gain relatif de la rentabilité. Ce gain relatif est des plus élevés en Afrique du Sud car l'effet du rendement a plus que compensé le surcoût en intrants associés au contrôle des ravageurs du cotonnier (semences + insecticides). Dans ce pays, l'adoption du CGM a en effet induit une augmentation du coût de la protection contre les ravageurs, ce qui est contraire aux affirmations couramment entendues. L'Afrique du Sud ne constitue pas un cas particulier dans ce domaine: dans beaucoup d'autres pays, l'on observe que l'augmentation du coût des semences dépasse l'économie réalisée sur les insecticides. La Chine fait exception, du moins dans la Vallée du Fleuve Jaune, car la réduction du nombre de traitements insecticides, à partir d'un nombre initial très élevé (fréquemment de 20 à 30), a été très forte. L'Australie semble aussi sortir du cas général depuis la réduction du coût que les producteurs de ce pays ont obtenue de Monsanto, à partir d'une baisse ${ }^{1}$ de la redevance d'emploi des semences.

\section{b - Le changement de structure des coûts de production, un risque financier accru}

La production cotonnière par le petit paysannat est peu intensive en Afrique du Sud. Les dépenses monétaires correspondent essentiellement à l'acquisition des semences et des insecticides. Dans ce pays, la distribution des semences se fait au

1 - Monsanto a consenti à baisser la redevance d'emploi de 245 à 170 dollars australiens, entre 1996-97 et 2001-02, de peur de voir les fermiers se détourner de ses variétés en l'absence de gains économiques. Avec le dernier niveau de la redevance, les fermiers doivent s'engager au respect strict des techniques d'utilisation du CGM. 
prix du marché depuis très longtemps. Le coût des semences de coton conventionnel se situait autour de 26 \$ ha et avait représenté 40-60\% du montant total du coût des intrants. L'adoption du CGM a fait passer ce taux à 70-80\%, comme le résultat des effets combinés d'une augmentation du prix des semences d'une part et de la réduction de la dépense en insecticides d'autre part (tableau 3, p. 817). C'est aussi le cas dans d'autres pays. La Chine fait encore exception, même si le passage au CGM a induit une forte augmentation du prix des semences ${ }^{2}$, car le niveau d'intensification est très élevé et le nombre de traitements insecticides reste assez élevé (tableau 5, p. 819).

L'augmentation de la part relative des semences dans le coût des intrants est globalement élevée. Pour les paysans à ressources financières limitées, le renchérissement des semences accroît le risque financier de la production car la dépense est consentie en début de cycle et elle ne peut être ajustée en fonction de l'évolution de la campagne (FOK et RAYMOND, 1995). La limitation ou la fragmentation de l'utilisation est une réponse à ce facteur risque. C'est d'ailleurs ce qui est observé en Afrique du Sud, où le sac de semences destiné à un hectare est utilisé pour ensemencer deux hectares ${ }^{3}$.

Cet aspect du changement dans la structure des coûts de production, et son incidence dans le risque financier perçu par les petits paysans, sont rarement soulignés dans les études d'impact du CGM.

\section{3 - Avantages et évolutions positives associés au CGM}

Une enquête menée pour cerner la perception des utilisateurs du CGM en Afrique du Sud fait ressortir l'avantage ressenti dans le contrôle des ravageurs (tableau 6, p. 819), en termes d'économie de coût, de travail et de réduction de la pénibilité du travail (GOUSE, KIRSTEN et JENKINS BEYERS, 2002). L'économie en coût d'insecticides est à entendre dans le sens large de réduction du coût d'acquisition des insecticides, avec d'importants coûts de transaction assumés par les paysans ${ }^{4}$. Au niveau des grands fermiers, le sentiment de confort dans le contrôle des ravageurs est mis en avant 5 et une évolution du complexe parasitaire serait perceptible: la réduction de l'emploi d'insecticides à base de pyréthrinoïdes aurait induit une plus grande présence d'insectes utiles (coccinelles). Cette perception doit être nuancée par l'observation d'une plus grande présence d'insectes piqueurs-suceurs (tels les pucerons et les jassides).

2 - Le prix a grosso modo triplé pour les variétés « population », et décuplé pour les variétés hybrides de CGM.

3 - Monsanto a dernièrement pris en compte ce comportement des paysans. Vis-à-vis des petits paysans, au lieu de distribuer des semences en sac de $25 \mathrm{~kg}$, il le fait avec des sacs de $5 \mathrm{~kg}$, mais le coût unitaire est $30 \%$ plus élevé que pour les semences distribués en sac de $25 \mathrm{~kg}$ dont les grands fermiers continuent à bénéficier.

4 - Se déplacer sur une grande distance pour aller acheter les produits insecticides, à un moment où les champs réclament leur présence, a un coût que le CGM permet de réduire.

5 - Le gros avantage évoqué correspond au sentiment de quiétude dans ce contrôle, dans la période entre Noël et Nouvel an. Les fermiers n'ont plus besoin de faire les comptages de ravageurs pour décider des bonnes dates des épandages ni de dépendre des conditions climatiques pour les réaliser. 


\section{III - UNE AGRICULTURE FRAGILE ET UNE MAUVAISE RENTABILITÉ EN ANNÉE DÉFAVORABLE}

Nous présentons ici les résultats de notre enquête pour replacer la production cotonnière du petit paysannat dans le contexte de l'agriculture des Makhatini Flats. Elle a été conduite dans huit villages des districts de Ubombo et de Ngwavuma dans une année où il n'y eut au total que 353 paysans à produire du coton. Les caractéristiques des exploitations ont été appréhendées à partir d'un échantillon de 193 fermes. Les perceptions des paysans sur la culture cotonnière ont été recueillies auprès de 86 exploitations et les coûts associés à cette culture ont été évalués auprès de 56 paysans. Les questionnaires ont été traduits en zoulou et les entretiens eurent lieu en trois phases, aux domiciles ou dans les champs des paysans. En dépit de la faible taille de l'échantillon, au regard du faible nombre de producteurs de coton en 2002-2003 (353 producteurs effectifs), la représentativité de l'enquête nous semble bonne.

\section{1 - L'Agriculture : une activité marginale en voie de disparition?}

Les familles des exploitations enquêtées sont de taille relativement grande, avec plus d'une douzaine de personnes, dont la moitié est composée d'enfants et de personnes âgées de plus de 55 ans (tableau 7, p. 820). Très peu de personnes sont signalées comme participant aux travaux dans les champs, cette situation explique que toutes les familles ressentent le besoin de disposer d'une maind'œuvre complémentaire qu'il faut rémunérer comptant.

La superficie disponible par exploitation est d'environ 7 ha, mais à peine plus du tiers fut effectivement mis en culture en 2002-2003. Cette sous-exploitation de la terre ne serait pas spécifique à cette campagne particulière. Elle traduit le manque de bras pour les travaux aux champs et elle témoigne du déclin de l'agriculture dans les Makhatini Flats. Beaucoup de responsables politiques en Afrique du Sud reconnaissent aujourd'hui qu'il est urgent et capital de redresser l'image négative de l'agriculture auprès des jeunes ruraux.

Les exploitations agricoles des populations autochtones sont assez fréquemment dirigées par une femme dont le taux de scolarisation est très bas. Plus de $80 \%$ des femmes chef d'exploitation n'ont pas été à l'école, et jamais pendant plus de six ans. Les hommes chef d'exploitation ne sont pas mieux lotis de ce point de vue.

\section{2 - Une spécialisation cotonnière peu typique}

Sur les 86 exploitations ayant initialement l'intention de cultiver du coton, 56 ont pu concrétiser cette culture, en y consacrant en moyenne 2,3 ha, soit la totalité des terres cultivées. C'est une spécialisation cotonnière peu typique en agriculture familiale, en Afrique. Au cours de cette campagne, nous n'avions pas vu d'autres espèces cultivées dans la zone d'enquête, indiquant que les paysans qui n'avaient pas pu cultiver le coton n'avaient rien cultivé en définitive. 
Le coton est cultivé pour le revenu qu'il peut générer : la sécurité de ce revenu est l'une des raisons les plus citées pour le cultiver, peu après l'avantage de disposer d'une assistance technique, même si cette assistance est très lâche comparée à ce qui se passe en Afrique francophone (tableau 8, p. 820). Ce sont des indications d'une agriculture en voie d'être abandonnée par les paysans et par ceux qui doivent donner l'appui nécessaire.

Comme dans la plupart des pays africains, on retrouve la même perception de la culture du coton, exigeante en travail et en intrants coûteux. Ce sont des contraintes que la disponibilité d'une rentrée financière régulière peut aider à surmonter, comme l'allocation de retraites aux personnes âgées ${ }^{6}$ : les exploitations cultivant effectivement le coton disposaient significativement de plus de ressources financières par cette voie (en moyenne, perception de 0,67 retraite par exploitation contre 0,17 ).

Les paysans manifestent une certaine connaissance des ravageurs du cotonnier : 80 \% d'entre eux déclarent bien les connaître. Ils ont été capables de citer, sur la base des photos montrées, les principaux ravageurs (les différentes chenilles des capsules et les pucerons essentiellement) ainsi que les prédateurs de ces ravageurs (les coccinelles et les larves de carabidés). Près de $70 \%$ d'entre eux avouent cependant avoir besoin de conseils de techniciens pour prendre la décision de réaliser les traitements insecticides.

\section{3 - Une faible intensification exacerbée en année défavorable}

La campagne 2002-2003 s'est caractérisée par une installation tardive des pluies et une sécheresse lors de la fructification du cotonnier. L'incertitude sur l'obtention de crédits pour acquérir les intrants, et qui a duré au-delà des dates normales de semis, a conduit à semer tardivement. Le retard enregistré pouvait être accentué par le retard dans la réalisation du labour pour lequel les paysans dépendent de la disponibilité des prestataires de service. L'enquête a permis de voir que tous les paysans ont fait réalisé ce labour en payant $370 \mathrm{Rd} / \mathrm{ha}(49,30 \$ / \mathrm{ha})$; c'est cher et peu justifié au regard du mauvais démarrage des pluies.

Les seuls intrants utilisés correspondent aux semences achetées et aux insecticides, pour un coût total de $75 \$$ ha pour les parcelles en coton $\mathrm{Bt}$, ce qui est significativement plus élevé que pour le coton conventionnel (61,30\$/ha). Dans le calcul de ce coût, nous avons intégré le fait que les paysans utilisaient un sac de semences ${ }^{7}$ pour ensemencer deux hectares alors qu'il était prévu plutôt pour un seul hectare : c'est une pratique qui a dû baisser le rendement dans les conditions climatiques de 2002-2003.

Le coût total pour le contrôle des ravageurs est plus faible que celui rapporté dans les études antérieures, car la faible pression parasitaire a induit un nombre

6 - En Afrique du Sud, une retraite mensuelle de 650 Rand (97 US\$ au cours de juin 2005, ou 53000 FCFA) est allouée aux femmes et aux hommes respectivement à partir de 60 ans et 65 ans.

7 - Le sac de $25 \mathrm{~kg}$ de semences était vendu à 387 Rand en 2002-03, auxquels il faut ajouter une redevance d'emploi de 350 Rand. Soit environ $98 \$$ le sac, ou 3,9 \$/kg. 
plus faible de traitements insecticides. En moyenne, les paysans ayant cultivé le coton conventionnel ont traité 2,5 fois contre les ravageurs du cotonnier. C'est certes significativement plus que dans le cas des paysans ayant adopté le coton $\mathrm{Bt}$, mais l'économie en traitement dans ce dernier cas ne représente même pas un traitement (tableau 9, p. 821). Il en résulte que le coût total du contrôle des ravageurs est significativement plus élevé avec le coton Bt. La part des semences de coton Bt dans le coût des intrants est de $65 \%$, du même ordre que celui obtenu dans les études antérieures.

Le coût du labour se révèle être du même niveau que celui des semences de coton Bt alors qu'il a dû induire une baisse de rendement, en accentuant le retard des semis. Il en découle que la part des semences dans les dépenses monétaires totales (sans cependant inclure la rémunération de la main-d'œuvre extérieure éventuelle ${ }^{8}$ ) est nettement plus faible.

La faible pression parasitaire déjà évoquée aurait pu permettre aux paysans cultivant le coton Bt de s'affranchir totalement de l'utilisation des insecticides à base de pyréthrinoïde (destinés au contrôle des chenilles). Ce n'est pas le cas, même si ces paysans en utilisent tout de même significativement moins. Les insectes piqueurs-suceurs ne peuvent pas être contrôlés par le coton Bt, l'utilisation d'insecticides à base d'organophosphorés peut être nécessaire et, dans la réalité, elle ne diffère pas significativement suivant le type de coton cultivé.

\section{4 - Une production cotonnière peu rentable voire déficitaire}

Dans les conditions climatiques défavorables de la campagne 2002-2003, le niveau de rendement atteint pour le coton conventionnel est proche de celui obtenu habituellement, de l'ordre de $400 \mathrm{~kg} / \mathrm{ha}$ de coton-graine. La dispersion autour de ce rendement est grande : près de $60 \%$ des paysans ne parviennent pas à atteindre ce rendement ( $30 \%$ dans le cas des paysans ayant adopté le coton $\mathrm{Bt}$ ). Le niveau de rendement du coton Bt est de l'ordre des rendements les plus bas enregistrés dans les travaux antérieurs, environ $500 \mathrm{~kg} / \mathrm{ha}$. Du fait de la grande dispersion des résultats de rendement consécutive aux conditions climatiques défavorables, mais aussi de la faiblesse de la taille de l'échantillon, la supériorité du rendement du coton Bt n'est pas significativement supérieure à celui du coton conventionnel (tableau 9, p. 821).

En considérant le revenu net des dépenses monétaires, et avant rémunération du travail familial, le coton $\mathrm{Bt}$ a rapporté en moyenne $84 \$$ ha, soit l'équivalent d'une allocation mensuelle pour personne âgée, ce qui n'est pas significativement supérieur au revenu net obtenu pour le coton conventionnel (58\$/ha). Même pour les années antérieures, qui n'ont pas souffert des mêmes difficultés climatiques et institutionnelles, on constate que le niveau de ce revenu net est faible par

8 - Nous n'avons pas pu saisir correctement cette information dans l'enquête. Nous pensons que cette dépense a dû être minime car les périodes de sécheresse laissaient le temps aux paysans d'entretenir les champs par eux-mêmes, alors que le regroupement de maturité des capsules réduisait l'exigence en main-d'œuvre à la récolte. 
rapport à l'allocation mensuelle de retraite (tableau 2, p. 817). L'incidence de la production cotonnière dans la réduction de la pauvreté paraît donc faible dans les Makhatini Flats.

L'analyse de la distribution des revenus éclaire sur la variation de la rentabilité et le caractère risqué de la culture cotonnière dans les conditions de la campagne 2002-2003. Environ 15 \% des paysans cultivant le coton conventionnel n'ont pas eu le revenu suffisant pour couvrir les dépenses monétaires consenties. Le fait d'utiliser le coton Bt n'a cependant pas accentué ce risque. Près des deux tiers des paysans ont obtenu un revenu net, certes positif, mais inférieur à $160 \$ /$ ha. Environ $70 \%$ des paysans ont produit avec un ratio output/input inférieur à deux, seuil généralement considéré comme minimal pour inciter les paysans ayant de faibles ressources financières à investir dans les intrants.

On devrait s'attendre à ce que les paysans décident de ne plus cultiver de coton ou d'abandonner le coton Bt. Une telle intention ne ressort pas de notre enquête à travers laquelle les paysans déclarent vouloir continuer à produire du coton et à utiliser le coton Bt, même s'ils se plaignent du coût élevé des semences. Cette intention est confirmée par le fait qu'environ 1500 paysans ont produit du coton dans la campagne 2003-2004, utilisant à $95 \%$ du coton Bt (les seules semences disponibles). Les paysans semblent ainsi faire la part des choses, tenant compte des conditions spécifiquement défavorables de la campagne 2002-2003 pour ne pas abandonner la culture cotonnière. Malheureusement, l'option privilégiant la production irriguée (voir supra) prive un grand nombre de paysans de la poursuite de cette culture.

\section{IV - LES LEÇONS POUR L'AFRIQUE ZONE FRANC}

L'Afrique du Sud a manifestement servi de tête de pont à la pénétration des OGM sur le continent africain. Le « succès » de l'expérience sud-africaine, dont on vient de voir le caractère nuancé, a fait l'objet d'une grande publicité auprès des autres pays africains. On n'a pas ménagé les efforts et les moyens pour faire connaître l'expérience des Makhatini Flats à des délégations de l'Afrique de l'Ouest, que l'on est parvenu à convaincre plus ou moins. L'analyse que nous venons de réaliser est fondée sur une approche plus globale, en s'appuyant par ailleurs sur un plus grand recul dans le temps et dans l'espace. Elle permet de tirer des enseignements plus complets et plus nuancés pour aider à la prise de décision sur l'adoption du CGM dans les pays de l'Afrique Zone Franc.

\section{1 - L'importance du facteur de stabilité institutionnelle}

La rentabilité de la production cotonnière dépend de la stabilité institutionnelle de la filière. Une introduction technologique, solution technique, ne peut contribuer à résoudre les problèmes d'ordre institutionnel. C'est pour cette raison que la production cotonnière en Afrique du Sud continue à décliner, de même que la part des petits paysans dans cette production. 
En situation d'instabilité, comme ce fut le cas de l'Afrique du Sud, la performance d'une introduction technologique s'en trouve réduite. Pis encore, cette introduction technologique peut avoir un effet défavorable pour accentuer une instabilité institutionnelle, ou pour l'orienter dans un sens discutable.

En conséquence, l'introduction du CGM est peu recommandable dans les pays de l'Afrique Zone Franc où la filière cotonnière est soumise à une instabilité institutionnelle. C'est le cas du Bénin, du Tchad, du Togo, de la Côte d'Ivoire, mais aussi de la Guinée, du Niger et de la Guinée Bissau, ces trois derniers pays ayant une production cotonnière devenue fort marginale. Une telle introduction n'est pas à recommander non plus pour les pays où l'évolution institutionnelle reste encore incertaine. C'est le cas du Mali et du Cameroun. Il ne reste donc que deux pays, le Burkina Faso et le Sénégal, où le contexte institutionnel est favorable à l'introduction du CGM.

\section{2 - La réorganisation nécessaire et coûteuse du système de distribution des semences}

Dans les pays où le CGM a été introduit, les semences sont distribuées délintées, traitées, conditionnées dans des sacs de 5 à $25 \mathrm{~kg}$, et surtout vendues au prix du marché. Le gain espéré dans la diffusion d'un produit biotechnologique s'ajoute en fait au gain issu de la distribution commerciale de semences. Le cas de l'Afrique du Sud est une illustration parfaite de ce scénario général.

Mais, de tous les pays de l'Afrique Zone Franc, seuls la Côte d'Ivoire, le Sénégal et le Burkina Faso ont réussi à distribuer des semences délintées. Le Mali n'a toujours pas réussi à concrétiser cet objectif formulé il y a plus de dix ans. Par ailleurs, seul le Burkina Faso a entrepris les actions nécessaires pour généraliser la distribution de semences délintées. Si le Burkina Faso paraît bien placé également pour ce critère relatif aux semences délintées, et à un degré moindre le Sénégal, pour tous les autres pays, se mettre à niveau supposera investissement et temps. Le temps ne sera pas seulement nécessaire pour réaliser les investissements, il le sera aussi pour accoutumer les paysans à l'usage d'une autre forme de semences, plus coûteuses, et à utiliser à dose plus faible.

Le surcoût ne résulte pas seulement du passage aux semences délintées, il découle aussi de la redevance d'emploi qu'il faudra acquitter pour utiliser un produit biotechnologique. L'Afrique du Sud a accepté de se plier aux conditions d'emploi imposées pratiquement dans tous les autres pays, dans des conditions coûteuses et pénalisantes pour les paysans à ressources limitées. Les pays de l'Afrique Zone Franc auront intérêt à se battre pour bénéficier de conditions qui leur soient plus adaptées, en s'inspirant en particulier de l'exemple de la Chine (FOK, LIANG, WANG et Wu, 2005). L'union fait la force dans le domaine de la négociation. La négociation de conditions sur une base régionale serait sans doute plus profitable, mais cette perspective est hypothéquée par la diversité du degré de stabilité institutionnelle ainsi que par la divergence de points de vue entre ces pays sur la pertinence du CGM.

L'adoption du CGM pose aussi le problème du partage du coût des semences. Ce problème ne s'est pas posé en Afrique du Sud dans la mesure où l'ancrage de 
l'orientation libérale de l'économie conduit naturellement à répercuter tout le coût aux paysans. L'Afrique Zone Franc a une autre tradition dans ce domaine, par ailleurs facteur du succès cotonnier qui a été enregistré (FOK, 2005). S'agissant des semences, les paysans ne paient pratiquement rien. Au Mali, depuis très longtemps, les paysans ne paient que le prix du sachet de produit de traitement des semences, soit environ 0,50 \$/ha. Même au Burkina Faso, avec une augmentation importante du coût de production de semences délintées, les paysans continuent à bénéficier d'un prix de cession très inférieur au coût réel. Il ne faut pas en conclure que les semences sont subventionnées (dans le sens d'une prise en charge extérieure et à perte). Il y a en fait un processus de soutien interprofessionnel de la filière, car le coût de production des semences est intégré dans les mécanismes de détermination du prix d'achat du coton-graine, de sorte que les paysans participent à ce coût réel, au-delà du prix qu'ils ont réellement payé lors de l'obtention des semences. C'est un exemple de processus de soutien à l'intensification intégré au mécanisme prix d'achat aux paysans dont l'efficacité a été soulignée (FOK et RAYMOND, 1995).

Comment préserver ce processus de soutien interprofessionnel dans l'utilisation de semences CGM est une question fondamentale à laquelle il faudra apporter une réponse efficace. Dans un contexte de promotion des semences d'ogm essentiellement guidée par des entités attirées par le profit qu'elles peuvent en tirer, on ne peut espérer qu'une réponse satisfaisante puisse prévaloir facilement.

\section{3 - L'accentuation du risque financier et les effets pervers}

La distribution des semences de CGM se traduit par un accroissement du poste de dépense en semences en début de saison. C'est le type de dépense pour lequel les paysans à ressources limitées manifestent le plus de réticence (FoK, 2002). À moins de parvenir à un mécanisme de partage des coûts qui n'entraîne pas une augmentation sensible de la dépense monétaire visible, les paysans peuvent changer leur comportement dans l'utilisation des semences avec des effets pervers regrettables (sous-dosage des semences lors du semis, instauration d'un trafic de semences, etc.) et dont les conséquences se répercutent à toutes les étapes des filières concernées.

Le contrôle du niveau de perception du risque financier, lié à l'utilisation des semences de CGM, est alors fondamental. Ce contrôle passe par la fixation du prix de cession des semences. Cela renvoie au point précédent de partage du coût, mais en intégrant peut-être d'autres acteurs que ceux directement concernés par les filières cotonnières.

\section{4 - La pondération de l'efficacité du coton Bt}

Le cas de l'Afrique du Sud confirme que l'utilisation du coton Bt est techniquement efficace mais que les effets sur la réduction de l'usage des insecticides et sur le rendement sont inversement proportionnels aux niveaux de cet usage et du 
rendement 9 (FOK, LIANG, WANG et Wu, 2005). Ce point est important car il signifie que, en moyenne, ce sont les paysans à niveau de rendement plus faible, pour des raisons liées aux contraintes structurelles de leurs exploitations, qui pourront espérer des niveaux plus élevés de gain relatif. C'est une particularité qui mérite d'être soulignée.

En partant d'un niveau de rendement plus élevé dans les pays de l'Afrique Zone Franc, avec un nombre de traitements plus élevé, mais dans des contextes de pression parasitaire quelque peu différents, les gains relatifs en rendement devraient être plus faibles et la réduction du nombre de traitements insecticides plus élevée en absolu. Un gain de rendement de $15 \%$ et une réduction de 2 à 3 traitements insecticides paraissent relever d'hypothèses plus réalistes que d'autres anticipations (CABANILla, ABDOUlAYE et SANDERSON, 2005 ; ElBEHRI et MACDONALD, 2004). Ces éléments peuvent servir de référence dans la négociation des conditions économiques d'utilisation des semences de coton Bt.

L'estimation précédente n'est bien sûr qu'indicative. Elle est proposée ici pour faire prendre conscience que l'acceptation des conditions économiques à l'usage d'une technologique doit être précédée d'une évaluation du gain qui peut en découler. S'agissant du CGM, beaucoup de pays ont eu à l'accepter sans une évaluation préalable correcte de son intérêt économique. Seuls des pays suffisamment puissants, avec des producteurs bien organisés (Australie), ont pu obtenir une révision à leur profit des conditions d'utilisation.

\section{5 - La gestion de la variabilité de la rentabilité, par une (difficile) cohabitation de CGM et de non-CGM}

Les résultats de notre recherche en Afrique du Sud ont montré la variabilité de la rentabilité entre les années d'une part et entre les producteurs une même année d'autre part. Pour les paysans dont la production est à faible rendement, l'utilisation du coton Bt n'a pas été justifiée ni rentable. En termes de conseil aux producteurs, on peut dire que, avant de semer, l'utilisation du coton Bt n'est pas pertinente si l'espérance de rendement au moment de semer n'est pas suffisamment élevée.

Beaucoup de facteurs influent sur l'espérance de rendement à un moment donné, tels que la date par rapport à la période optimale de semis, la pluviosité qui avait précédé, la situation de la main-d'œuvre et l'équipement du paysan, etc. La grande diversité des niveaux de rendement observés et des pratiques paysannes s'explique par le comportement des paysans, ajustant leurs pratiques aux espérances de rendement (FOK, KONÉ, DJOUARA et BALLO, 1999). Une expression flagrante de ce type de comportement est l'abandon des parcelles en cours de culture.

Dans le contexte agricole des pays de l'Afrique Zone Franc, il y aura toujours une fraction notable de parcelles cotonnières installées dans des conditions

9 - L'utilisation du coton Bt permet de réduire le manque à gagner lié au contrôle chimique contre les ravageurs. Moins ce contrôle est optimal, comme c'est le cas avec les paysans appliquant peu et mal les insecticides, plus le manque à gagner est grand et plus l'effet du coton Bt pour récupérer le manque à gagner est important (FOK et al., 2005). 
induisant une faible espérance de rendement et pour lesquelles il ne sera pas justifié d'utiliser le coton Bt. Cette analyse rectifie ainsi le débat sur les ogm qui repose implicitement, de la part des partisans comme des opposants aux OGM, sur une couverture en OGM maximale, voire à $100 \%$. Notre analyse indique qu'une utilisation efficace et durable des OGM doit procéder de leur cohabitation ${ }^{10}$ avec des variétés non-OGM, dans le respect de la flexibilité des prises de décision des producteurs en fonction des conditions externes et internes à leurs exploitations.

La gestion d'une distribution des semences dans l'esprit d'une cohabitation induit des contraintes supplémentaires, à forte incidence sur le fonctionnement pratique des filières concernées, mais dont l'explicitation nous entraînerait trop loin. Cette gestion requiert une bonne stabilité institutionnelle des filières et une réelle entente entre les acteurs. Elle s'oppose aussi aux appétits des vendeurs de semences et de technologie qui y verraient une réduction de leur marché.

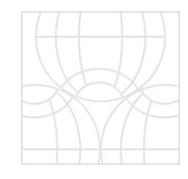

La production cotonnière en Afrique du Sud est d'abord celle des fermiers blancs. Si l'on considère que l'utilisation du CGM est un succès dans ce pays, c'est d'abord le cas d'un succès d'utilisation par ces fermiers.

Avec une production fluctuante voire déclinante, le succès du CGM est à nuancer. Le cas de l'Afrique du Sud montre que l'introduction d'une technologie nouvelle ne suffit pas pour assurer la progression de la production à l'échelle du pays. D'autres facteurs d'ordre institutionnel interviennent : il est peu réaliste de penser qu'une solution technique puisse résoudre les problèmes d'ordre institutionnel. Pis encore, le fait de se focaliser sur une solution technique a certainement contribué à détourner l'attention des problèmes institutionnels à résoudre.

La filière cotonnière de l'Afrique du Sud a connu une évolution institutionnelle d'une instabilité exacerbée au cours des dernières années et elle se répercute surtout sur la production des petits paysans. La dernière étape de cette évolution peut être considérée comme un changement dans le mode de production, orientée vers une intensification sécurisée par l'irrigation. C'est une étape qui exprime un phénomène de forclusion du droit d'usage de la terre par les petits paysans concernés. Elle peut être perçue aussi comme une option de prolétarisation d'un nombre limité de paysans en laissant pour compte la plus grande masse, du moins si elle ne peut être étendue à plus grande échelle dans une région où l'agriculture est en voie de disparaitre. Cette évolution de la production cotonnière et de l'agriculture dans son ensemble, bien plus que la seule question technique du CGM, nous semble devoir interpeller davantage les pouvoirs publics et la société civile.

10 - Nous retenons sciemment ce vocable de cohabitation et non de coexistence, car ce dernier terme est très connoté par rapport aux préoccupations environnementales et au respect des filières de production de type organique ou biologique. 
Sans remettre en cause les résultats antérieurs sur l'adoption du CGM en Afrique du Sud, nos travaux mettent en évidence une mauvaise rentabilité dans une année défavorable sur le plan climatique et institutionnel. Ce résultat rappelle que l'agriculture pluviale reste une activité sensible aux aléas climatiques et que l'introduction d'une technologie nouvelle peut accroître le risque financier associé à son adoption.

Globalement cependant, en tenant compte de l'ensemble des travaux en Afrique du Sud, la rentabilité de l'adoption du CGM est assez fluctuante à un niveau très faible de rendement. L'adoption du CGM induit un changement important dans la structure des coûts de production, le poste des semences devenant l'élément principal des dépenses monétaires. C'est une évolution qui n'est pas compatible avec l'aversion au risque des paysans dont les ressources financières sont limitées.

Le degré d'instabilité ou d'incertitude institutionnelle dans la plupart des pays de l'Afrique Zone Franc, à l'exception du Burkina Faso et du Sénégal, n'offre pas de contexte favorable à l'introduction du CGM. La distribution des semences de CGM se fait en général suivant une démarche et des principes économiques prônés en Afrique Zone Franc par des experts (THE Michell Group INC., 2004), mais opposés à ceux qui y ont toujours prévalu. La diffusion du CGM devra donc se traduire par une réorganisation de la distribution de semences, exigeante en investissement et en temps mais aussi en innovation institutionnelle, pour permettre un partage des coûts favorables aux paysans. Une telle innovation ne coule pas de source en raison de la motivation de profit rapide et important associé au transfert biotechnologique.

Dans les PVD à faible productivité agricole, c'est un faible coût d'utilisation des semences de CGM qui conditionne la rentabilité de celles-ci. Cela suppose de négocier et de refuser les conditions habituellement imposées par les fournisseurs de CGM. L'analyse de l'expérience de l'Afrique du Sud indique aussi que l'adoption du CGM ne signifie pas l'utilisation exclusive. La prise en compte des contraintes des paysans en Afrique milite au contraire pour une utilisation conjointe avec des variétés conventionnelles. Une telle cohabitation alourdit le cahier des charges de la nécessaire réorganisation de la distribution des semences.

Toutes les difficultés soulignées ici, jamais évoquées par ailleurs, mettent en garde contre une décision précipitée dans la diffusion du CGM dans les pays de l'Afrique Zone Franc. 


\section{ANNEXES}

Tableau 1 : Faible contribution du petit paysannat à la production cotonnière en Afrique du Sud

(Short smallholders' contribution to cotton production)

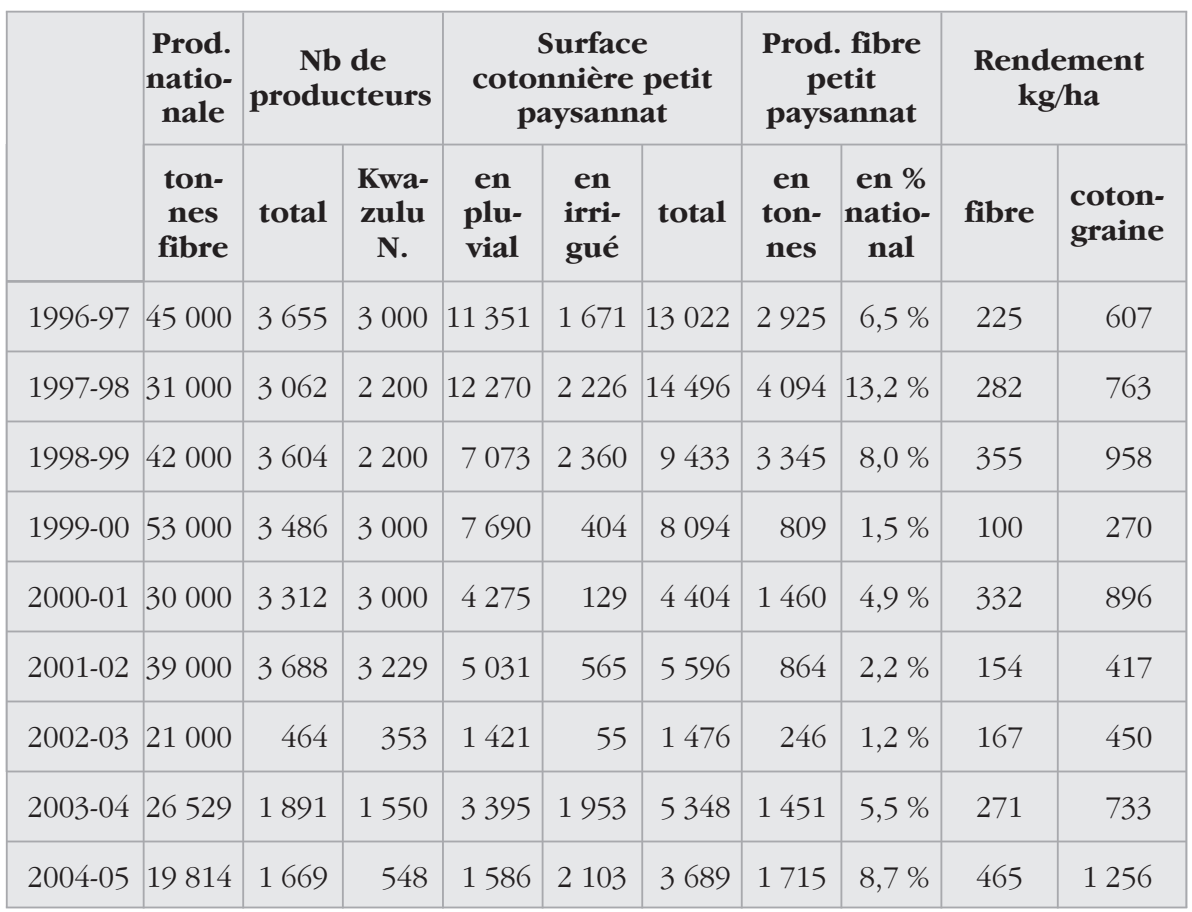

Note : Nous n'avons pas comptabilisé les paysans produisant sous contrat avec l'entreprise Makhatini Gins dans le petit paysannat.

Sources : Cotton SA, Small-scale Farmer Development. 
Tableau 2 : Synthèse des travaux d'évaluation de la rentabilité de l'adoption du CGM par le petit paysannat en Afrique du Sud

(Synthesis on the profitability assessment studies of the GMC adoption by smallholders in South Africa)

\begin{tabular}{|c|c|c|c|c|c|c|c|c|c|}
\hline \multirow[t]{2}{*}{ Source } & \multirow[t]{2}{*}{$\begin{array}{l}\text { Campa- } \\
\text { gne }\end{array}$} & \multicolumn{2}{|c|}{$\begin{array}{l}\text { Rendement } \\
\text { coton- } \\
\text { graine }\end{array}$} & \multicolumn{2}{|c|}{$\begin{array}{c}\text { Marge } \\
\text { nette, } \$ / \text { ha }\end{array}$} & \multicolumn{4}{|c|}{$\begin{array}{l}\text { Variation due au coton } \mathrm{Bt}(\% \\
\text { coton non-Bt) }\end{array}$} \\
\hline & & $\begin{array}{c}\text { Coton } \\
\text { Bt }\end{array}$ & $\begin{array}{c}\text { Coton } \\
\text { non- } \\
\text { Bt }\end{array}$ & $\begin{array}{c}\text { Coton } \\
\text { Bt }\end{array}$ & $\begin{array}{c}\text { Coton } \\
\text { non- } \\
\text { Bt }\end{array}$ & $\begin{array}{c}\text { Rende- } \\
\text { ment }\end{array}$ & $\begin{array}{l}\text { Coût } \\
\text { in- } \\
\text { trants }\end{array}$ & $\begin{array}{l}\text { Coût } \\
\text { moné- } \\
\text { taire }\end{array}$ & $\begin{array}{r}\text { Marge } \\
\text { nette }\end{array}$ \\
\hline \multirow{3}{*}{$\begin{array}{l}\text { MORSE, } \\
\text { BEN- } \\
\text { NETT et } \\
\text { al. } \\
2004\end{array}$} & 1998-99 & 738 & 452 & 167 & 81 & $63 \%$ & $20 \%$ & $19 \%$ & $106 \%$ \\
\hline & 1999-00 & 489 & 264 & 57 & -2 & $85 \%$ & $25 \%$ & $17 \%$ & \\
\hline & 2000-01 & 783 & 501 & 136 & 43 & $56 \%$ & $-22 \%$ & $-17 \%$ & $216 \%$ \\
\hline $\begin{array}{l}\text { KIRS- } \\
\text { TEN, } \\
\text { GOUSE } \\
\text { et } \\
\text { BEYERS, } \\
2002\end{array}$ & 1999-00 & 576 & 395 & 184 & 128 & $46 \%$ & $51 \%$ & & $43 \%$ \\
\hline \multirow{2}{*}{$\begin{array}{l}\text { THIRTLE } \\
\text { et al., } \\
2003\end{array}$} & $1998-99$ & 475 & 457 & 126 & 128 & $4 \%$ & $16 \%$ & & $-1 \%$ \\
\hline & 1999-00 & 425 & 304 & 103 & 66 & $40 \%$ & $16 \%$ & & $56 \%$ \\
\hline
\end{tabular}

Tableau 3 : Augmentation de la part des semences dans les coûts de production en conséquence de 1'utilisation du CGM en Afrique du Sud (Increase of the seed share in production cost as consequence of GMC use by smallholders in South Africa)

\begin{tabular}{|c|c|c|c|c|c|}
\hline \multirow[b]{2}{*}{ Source } & \multirow[b]{2}{*}{ Campagne } & \multicolumn{2}{|c|}{ Coton Bt } & \multicolumn{2}{|c|}{ Coton non-Bt } \\
\hline & & $\begin{array}{l}\text { \% coût des } \\
\text { intrants }\end{array}$ & $\begin{array}{l}\text { \% coût } \\
\text { monétaire }\end{array}$ & $\begin{array}{l}\text { \% coût des } \\
\text { intrants }\end{array}$ & $\begin{array}{l}\text { \% coût } \\
\text { monétaire }\end{array}$ \\
\hline \multirow{3}{*}{$\begin{array}{l}\text { MORSE, } \\
\text { BENNETT et } \\
\text { al. , } 2004\end{array}$} & 1998-99 & $79 \%$ & $49 \%$ & $47 \%$ & $29 \%$ \\
\hline & 1999-00 & $80 \%$ & $60 \%$ & $46 \%$ & $32 \%$ \\
\hline & 2000-01 & $69 \%$ & $42 \%$ & $36 \%$ & $24 \%$ \\
\hline $\begin{array}{l}\text { KIRSTEN, } \\
\text { GOUSE et } \\
\text { BEYERS, } \\
2002\end{array}$ & 1999-00 & $74 \%$ & & $49 \%$ & \\
\hline \multirow{2}{*}{$\begin{array}{l}\text { THIRTLE et } \\
\text { al., } 2003\end{array}$} & 1998-99 & $68 \%$ & & $47 \%$ & \\
\hline & 1999-00 & $72 \%$ & & $50 \%$ & \\
\hline
\end{tabular}


Tableau 4 : Impacts variables du cotton Bt dans le monde (Diversified impacts of Bt-Cotton use in the world)

\begin{tabular}{|c|c|c|c|c|c|c|c|c|c|c|}
\hline \multirow[t]{2}{*}{ Pays } & \multirow[t]{2}{*}{ Source } & \multirow[t]{2}{*}{$\begin{array}{l}\text { Campa- } \\
\text { gne }\end{array}$} & \multicolumn{2}{|c|}{$\begin{array}{l}\text { Rendt } \\
\text { coton- } \\
\text { graine }\end{array}$} & \multicolumn{2}{|c|}{$\begin{array}{l}\text { Marge } \\
\text { nette, } \$ / \text { ha }\end{array}$} & \multicolumn{4}{|c|}{$\begin{array}{l}\text { Variation due au cotton } \mathrm{Bt} \\
(\% \text { coton non-Bt) }\end{array}$} \\
\hline & & & $\begin{array}{l}\text { Coton } \\
\text { Bt }\end{array}$ & $\begin{array}{l}\text { Coton } \\
\text { non- } \\
\text { Bt }\end{array}$ & $\begin{array}{l}\text { Coton } \\
\text { Bt }\end{array}$ & $\begin{array}{l}\text { Coton } \\
\text { non- } \\
\text { Bt }\end{array}$ & Rendt & $\begin{array}{l}\text { Coût } \\
\text { in- } \\
\text { trants }\end{array}$ & $\begin{array}{l}\text { Coût } \\
\text { moné- } \\
\text { taire }\end{array}$ & $\begin{array}{l}\text { Marge } \\
\text { nette }\end{array}$ \\
\hline \multirow[t]{4}{*}{ Chine } & \multirow{3}{*}{$\begin{array}{l}\text { PRAY et } \\
\text { al., } 2002\end{array}$} & 1999-00 & 3371 & 3186 & 967 & 749 & $6 \%$ & & $-23 \%$ & $29 \%$ \\
\hline & & 2000-01 & 2941 & 1901 & 1208 & 658 & $55 \%$ & & $4 \%$ & $84 \%$ \\
\hline & & 2001-02 & 3481 & 3138 & 833 & 621 & $11 \%$ & & $-17 \%$ & $34 \%$ \\
\hline & $\begin{array}{l}\text { Xu et al. } \\
2004^{*}\end{array}$ & 2002-03 & 3615 & 3829 & 1824 & 1963 & $-6 \%$ & $43 \%$ & & $-7 \%$ \\
\hline \multirow[t]{4}{*}{ Inde } & \multirow{2}{*}{$\begin{array}{l}\text { BENETT } \\
\text { et al., } \\
2004^{*}\end{array}$} & $2002-03$ & 2180 & 1500 & 808 & 542 & $45 \%$ & $15 \%$ & & $49 \%$ \\
\hline & & 2003-04 & 2250 & 1380 & 1106 & 636 & $63 \%$ & $2 \%$ & & $74 \%$ \\
\hline & \multirow[t]{2}{*}{$\begin{array}{l}\text { ORPHAL, } \\
2005\end{array}$} & $\begin{array}{l}\text { 2002-03 } \\
\text { non- } \\
\text { irrigué }\end{array}$ & 1253 & 1093 & 270 & 339 & $15 \%$ & $107 \%$ & $26 \%$ & $-20 \%$ \\
\hline & & $\begin{array}{l}\text { 2002-03 } \\
\text { irrigué }\end{array}$ & 1683 & 1556 & 475 & 359 & $8 \%$ & $35 \%$ & $8 \%$ & $32 \%$ \\
\hline \multirow[t]{3}{*}{$\begin{array}{l}\text { Mexi- } \\
\text { que }\end{array}$} & $\begin{array}{l}\text { MAGAÑA } \\
\text { et al., } \\
1999 * *\end{array}$ & $1998-99$ & 2068 & 1704 & 1102 & 630 & $21 \%$ & $-5 \%$ & & $75 \%$ \\
\hline & \multirow{2}{*}{$\begin{array}{l}\text { TRAXLER } \\
\text { et al., } \\
2003 \text { et } \\
\text { FAO, } \\
2004\end{array}$} & $1997-98$ & 2197 & 2197 & & & $0 \%$ & & & \\
\hline & & 1998-99 & 4957 & 4142 & & & $20 \%$ & & & \\
\hline \multirow[t]{2}{*}{$\begin{array}{l}\text { Argen- } \\
\text { tine }\end{array}$} & \multirow{2}{*}{$\begin{array}{l}\text { QAIM et } \\
\text { DE JAN- } \\
\text { VRY, } \\
2003\end{array}$} & 1999-00 & 2062 & 1558 & 174 & 135 & $32 \%$ & & & $29 \%$ \\
\hline & & 2000-01 & 2182 & 1625 & 19 & 12 & $34 \%$ & & & $58 \%$ \\
\hline \multirow{6}{*}{$\begin{array}{l}\text { Austra- } \\
\text { lie }\end{array}$} & \multirow{6}{*}{$\begin{array}{l}\text { FITT, } \\
2003 * *\end{array}$} & $1996-97$ & 4613 & 4929 & & & $-6 \%$ & $9 \%$ & & \\
\hline & & $1997-98$ & 5026 & 5002 & & & $0 \%$ & $8 \%$ & & \\
\hline & & 1998-99 & 4076 & 4410 & & & $-8 \%$ & $-12 \%$ & & \\
\hline & & 1999-00 & 4805 & 4763 & & & $1 \%$ & $-13 \%$ & & \\
\hline & & 2000-01 & 4529 & 4381 & & & $3 \%$ & $-30 \%$ & & \\
\hline & & 2001-02 & 5497 & 5234 & & & $5 \%$ & $-35 \%$ & & \\
\hline \multirow[t]{2}{*}{ USA } & \multirow{2}{*}{$\begin{array}{l}\text { FALCK- } \\
\text { ZEPEDA } \\
\text { et al., } \\
1999^{* *}\end{array}$} & 1998-99 & $\begin{array}{l}1270 \\
\text { à } \\
2540\end{array}$ & & & & $\begin{array}{l}3 \text { à } \\
11 \%\end{array}$ & $\begin{array}{l}-6 \text { à } \\
-18 \%\end{array}$ & & \\
\hline & & $\begin{array}{l}1996 \text { à } \\
2000\end{array}$ & 2748 & 2550 & & & $8 \%$ & & & \\
\hline
\end{tabular}

* Marge nette avec seulement une déduction du coût des semences et des insecticides.

** Variation du coût des intrants en tenant compte seulement du poste de contrôle des ravageurs (semences + insecticides). 
Tableau 5 : Changement de la structure des coûts de production lié à l'utilisation du coton Bt en dehors de l'Afrique du Sud

(Change in the production cost structure resulting from using Bt-Cotton out of South Africa)

\begin{tabular}{|c|c|c|c|c|c|c|}
\hline \multirow[t]{2}{*}{ Pays } & \multirow[t]{2}{*}{ Source } & \multirow[t]{2}{*}{ Campagne } & \multicolumn{2}{|c|}{ Coton Bt } & \multicolumn{2}{|c|}{ Coton non-Bt } \\
\hline & & & $\begin{array}{l}\text { \% coût } \\
\text { des } \\
\text { intrants }\end{array}$ & $\begin{array}{l}\text { \% coût } \\
\text { moné- } \\
\text { taire }\end{array}$ & $\begin{array}{l}\text { \% coût } \\
\text { des } \\
\text { intrants }\end{array}$ & $\begin{array}{c}\text { \% coût } \\
\text { moné- } \\
\text { taire }\end{array}$ \\
\hline \multirow[t]{3}{*}{ Chine } & \multirow{3}{*}{$\begin{array}{l}\text { PRAY et al., } \\
2002\end{array}$} & 1999-00 & $23 \%$ & $16 \%$ & $15 \%$ & $12 \%$ \\
\hline & & 2000-01 & $21 \%$ & $16 \%$ & $7 \%$ & $6 \%$ \\
\hline & & 2001-02 & $22 \%$ & $18 \%$ & $4 \%$ & $3 \%$ \\
\hline \multirow[t]{2}{*}{ Inde } & \multirow[t]{2}{*}{$\begin{array}{l}\text { ORPHAL, } \\
2005\end{array}$} & $\begin{array}{l}\text { 2002-03 } \\
\text { non- } \\
\text { irrigué }\end{array}$ & $74 \%$ & $34 \%$ & $38 \%$ & $11 \%$ \\
\hline & & $\begin{array}{l}\text { 2002-03 } \\
\text { irrigué }\end{array}$ & $79 \%$ & $31 \%$ & $26 \%$ & $8 \%$ \\
\hline Mexique & $\begin{array}{l}\text { MAGAÑA et } \\
\text { al., 1999** }\end{array}$ & 1998-99 & $38 \%$ & & $12 \%$ & \\
\hline \multirow[t]{2}{*}{ Argentine } & \multirow{2}{*}{$\begin{array}{l}\text { QAIN et DE } \\
\text { JANVRY, } \\
2003\end{array}$} & 1999-00 & $60 \%$ & $29 \%$ & $15 \%$ & $5 \%$ \\
\hline & & 2000-01 & $55 \%$ & $26 \%$ & $16 \%$ & $6 \%$ \\
\hline
\end{tabular}

Tableau 6 : Répartition des paysans en fonction des avantages perçus du coton $\mathrm{Bt}$ (Distribution of the smallholders accoridng to the advantages associated to using Bt-cotton)

\begin{tabular}{|l|c|c|}
\hline \multirow{2}{*}{\multicolumn{1}{|c|}{ Avantages perçus }} & \multicolumn{2}{|c|}{ Pourcentage des paysans utilisant } \\
\cline { 2 - 3 } & Coton conventionnel & Coton Bt \\
\hline Augmentation du rendement & $62 \%$ & $58 \%$ \\
\hline Meilleure qualité du coton & $12 \%$ & $30 \%$ \\
\hline Prix plus élevé du coton & $12 \%$ & $15 \%$ \\
\hline Économie en pesticides & $77 \%$ & $70 \%$ \\
\hline Économie en travail & $42 \%$ & $35 \%$ \\
\hline Moins de traitements pesticides & $30 \%$ & $18 \%$ \\
\hline Autres & $27 \%$ & $40 \%$ \\
\hline
\end{tabular}


Tableau 7 : Structure et contraintes des exploitations du petit paysannat (écart-type entre parenthèses)

(Farm smallholding Structure and constraints)

\begin{tabular}{|l|c|c|c|}
\hline & \multicolumn{2}{|c|}{ Chef d'exploitation est } & \multirow{2}{*}{ Total } \\
\hline & femme & homme & \\
\hline Nombre d'exploitations & 78 & 115 & 192 \\
\hline $\begin{array}{l}\text { \% chefs d'exploitation sans } \\
\text { scolarisation }\end{array}$ & $82,7 \%$ & $83,6 \%$ & \multirow{2}{*}{$83,5 \%$} \\
\hline $\begin{array}{l}\text { Nb de personnes dans l'exploi- } \\
\text { tation* }\end{array}$ & $13,4(5,5)$ & $11,8(4,9)$ & 12,6 \\
\hline Nb de personnes > 56 ans & 2,2 & 2 & 2,1 \\
\hline $\begin{array}{l}\text { Nb de personnes < } 16 \text { ans } \\
\text { \% de paysans ressentant le } \\
\text { besoin en main-d'œuvre } \\
\text { complémentaire }\end{array}$ & 4,9 & 4,6 & 4,9 \\
\hline $\begin{array}{l}\text { Surface totale disponible par } \\
\text { exploitation (ha) }\end{array}$ & $100 \%$ & $100 \%$ & $100 \%$ \\
\hline $\begin{array}{l}\text { Surface cultivée par exploita- } \\
\text { tion (ha) }\end{array}$ & $7,4(7,8)$ & $6,2(3,8)$ & 6,7 \\
\hline $\begin{array}{l}\text { Ratio d'usage de la terre dispo- } \\
\text { nible }\end{array}$ & $35 \%$ & $1,8(2,1)$ & 2,1 \\
\hline
\end{tabular}

* Différence significative

Tableau 8 : Distribution des raisons données par les paysans en faveur de la culture du coton

(Distribution of the reasons farmers give in favour of growing cotton)

\begin{tabular}{|l|r|r|r|}
\hline \multirow{2}{*}{ Raisons pour cultiver le coton } & \multicolumn{2}{|c|}{$\begin{array}{c}\text { Nombre de réponses } \\
\text { au rang }\end{array}$} & $\begin{array}{c}\text { Nombre total } \\
\text { de réponses }\end{array}$ \\
\cline { 2 - 3 } & $\mathbf{1}$ & $\mathbf{2}$ & 62 \\
\hline Revenu monétaire sûr & 37 & 7 & 26 \\
\hline Paiement rapide & 7 & 12 & 20 \\
\hline Rentabilité & 3 & 9 & 34 \\
\hline Crédit intrant obtenu & 3 & 6 & 74 \\
\hline Appui technique obtenu & 6 & 41 & 58 \\
\hline Culture adaptée localement & 29 & 11 & 2 \\
\hline Autres & & & 276 \\
\hline Total & 85 & 86 & \\
\hline
\end{tabular}


Tableau 9 : Coûts de production et rentabilité du coton en 2002-2003

(écart-type entre parenthèses)

(Production cost and profitability in 2002-2003)

\begin{tabular}{|c|c|c|c|c|}
\hline & \multicolumn{2}{|c|}{ Type de coton } & \multicolumn{2}{|c|}{$\begin{array}{l}\text { Différence (Bt- } \\
\text { conventionnel) }\end{array}$} \\
\hline & Bt & Conventionnel & En valeur & En \% \\
\hline $\begin{array}{l}\text { Nb moyen de traitements } \\
\text { insecticides }\end{array}$ & $1,9(0,6)$ & $2,5(0,8)$ & $-0,6^{*}$ & -24 \\
\hline $\begin{array}{l}\% \text { de paysans à utiliser cyper- } \\
\text { méthrine }\end{array}$ & $60 \%$ & $84,60 \%$ & $-24,60 \%$ & \\
\hline $\begin{array}{l}\text { Dose de cyperméthrine g/ha } \\
\text { (contre chenilles) }\end{array}$ & $12,4(12,2)$ & $24,2(15,0)$ & $-11,8^{*}$ & $-49 \%$ \\
\hline $\begin{array}{l}\text { Dose de Monostem g/ha } \\
\text { (contre piqueurs-suceurs) }\end{array}$ & $110,0(61,0)$ & $88,0(52,0)$ & 22 & $25 \%$ \\
\hline Coût des insecticides $(\$ / \mathrm{ha})$ & $25,9(12,4)$ & $35,6(14,5)$ & $-9,7 *$ & $-27 \%$ \\
\hline Coût des semences $(\$ /$ ha) & 49,0 & 25,7 & $23,3^{*}$ & $91 \%$ \\
\hline Coût total des intrants $(\$ / h a)$ & $75,0(12,4)$ & $61,3(14,5)$ & $13,7^{*}$ & $22 \%$ \\
\hline Coût intrants + labour $(\$ / h a)$ & $124,4(124,4)$ & $110,6(110,6)$ & $13,8^{*}$ & $12 \%$ \\
\hline Ratio coût semences/intrants & $65,3 \%$ & $41,9 \%$ & $23,4 \%$ & \\
\hline $\begin{array}{l}\text { Ratio coût semences/ } \\
\text { dépenses monétaires** }\end{array}$ & $39,4 \%$ & $23,2 \%$ & $16,2 \%$ & \\
\hline $\begin{array}{l}\text { Rendement coton-graine (kg/ } \\
\text { ha) }\end{array}$ & $522(241)$ & $423(259)$ & 99 & $23 \%$ \\
\hline $\begin{array}{l}\text { \% des exploitations à moins } \\
\text { de } 400 \mathrm{~kg} \text { /ha }\end{array}$ & $30,00 \%$ & $57,7 \%$ & $-27,7 \%$ & \\
\hline $\begin{array}{l}\text { Revenu net des dépenses } \\
\text { monétaires }(\$ / \mathrm{ha})\end{array}$ & $84(97)$ & $58(107)$ & 26 & $45 \%$ \\
\hline $\begin{array}{l}\% \text { des exploitations à revenu } \\
\text { net négatif }\end{array}$ & $6,7 \%$ & $15,4 \%$ & $-8,7 \%$ & \\
\hline $\begin{array}{l}\text { \% des exploitations à revenu } \\
\text { net de } 0 \text { à } 160 \$ \text { ha }\end{array}$ & $63,3 \%$ & $57,7 \%$ & $5,6 \%$ & \\
\hline $\begin{array}{l}\% \text { des exploitations à ratio } \\
\text { output/input }<2\end{array}$ & $73,4 \%$ & $69,9 \%$ & $3,8 \%$ & \\
\hline
\end{tabular}

* Test de Student significatif à $5 \%$

** Dépenses monétaires sans la main-d'œuvre rémunérée 


\section{BIBLIOGRAPHIE}

BeNNETT R., ISMAËL Y., KAMBHAMPATI U. et Morse S., 2004, «Economic impact of genetically modified cotton in India », AgBioForum 7, 3, pp. 96-100.

Cabanilla l.S., Abdoulaye T. et SanDERSON H.J., 2005, « Economic cost of non-adoption of Bt Cotton in Africa: with special reference to Mali ", International Journal of Biotechnology 7 , 1/2/3, pp. 46-61.

EDENS E.R., SLINSKY S., LARSON J.A., ROBERTS R.K. et LENTZ G.L., 1998, «Economic analysis of genetically engineered Bt cotton for tobacco budworm and bollworm control», in Beltwide Cotton Conferences, pp. 380-383.

Elbehri A. et MacDonald S., 2004, "Estimating the Impact of Transgenic Bt Cotton on West and Central Africa: Ageneral Equilibrum Approach », World Development 32, 12, pp. 2049-2064.

FAO, 2004, "La situation mondiale de l'alimentation et de l'agriculture 20032004. Les biotechnologies agricoles: une réponse aux besoins des plus démunis?», Collection Agriculture, $\mathrm{N}^{\circ}$ 35, FAO, Rome, p. 227.

FitT G.P., 2003, «Utilisation et impact des cotons Bt transgéniques en Australie», ICAC recorder, décembre 2003, pp. 16-22.

FoK A.C.M., 2002, Cotton future in Western and Central Africa: the challenge of combining technical and institutional innovations, OCL 9, 2-3, pp. 115 122.

FoK A.C.M., 2005, « Libéralisation, distorsion de concurrence et évolution technologique: portée et limites du succès du coton en Afrique Zone Franc», in International Conference on Championing Agricultural Successes for Africa's Future. A Parliamenta- rians' Dialogue on NEPAD Omerset West, South Africa, 15-18 May 2006.

FoK A.C.M., LIANG W., WANG G. et WU Y., 2005, « Diffusion du coton génétiquement modifié en Chine: leçons sur les facteurs et limites d'un succès ", Économie Rurale, 285, pp. 5-32.

FoK A.C.M. et RAYMOND G., 1995, «Organisation locale d'adaptation au marché mondial du coton : le cas du Mali », in Calabre S. (ed), Matières premières, marchés mondiaux, déséquilibres, organisation, pp. 203-214, Ministère de la Coopération, Economica, Paris.

FoK A.C.M., Kone M., DJOUARA H. et Ballo D., 1999, «Diversité des pratiques paysannes en zones cotonnières du Mali: portée et limites des gestions d'itinéraires techniques observées ", in Rôle et place de la recherche pour le développement des filières cotonnières en évolution en Afrique Montpellier, sept. 1-2, 1999, pp. $137-$ 159, CIRAD.

Gouse M., Kirsten J. et Jenkins BEYERS L., 2002, «Bt cotton in South Africa: adoption and the impact on farm incomes amongst small-scale and large scale farmers », Working paper, p. 13, University of Pretoria, Department of Agricultural Econmics, Pretoria.

Gouse M., Kirsten J., Shankar B. et THIRTLE C., 2005, Bt cotton in Kwazulu Natal: technological Triumph but institutional failure, www.agbiotechnet.com

Grassi (DE) A. , 2003, «Genetically Modified Crops and sustainable poverty alleviation in Sub-Saharan Africa. An assessment of current evidence ", Third World Network - Africa, p. 92. 
HOAG H., 2003, «Biotech firms join charities in drive to help Africa's farms ", Nature, 422, 246, p. 1.

Ismä̈l Y., Beyers L., Thirtle C. et Piesse J., 2002, « Efficiency effects of Bt cotton. Smallholder adoption and economic impacts of Bt cotton in Makhathini Flats, KwaZulu Natal, South Africa », in EVENSON R.E., SANTANIELLO V. et Zilberman D. (eds), Economic and Social Issues in Agricultural Biotechnology, pp. 325-349, CABI Publishing, Wallington and New York.

KeRBY T.A., 2001, "What changes in yield and quality are due to transgenic varieties? ", The cotton gin and oil mill press, pp. 5-7.

MACrae J., 1995, La compétitivité du coton dans le monde Pays hors zone franc, Ministère de la Coopération, Paris, p. 321.

MANÉ D., 2005, «Adoption du coton transgénique au Burkina Faso : des pas de géant pour l'envol de l'or blanc ", in Le Soleil mulitmedia, Dakar (Sénégal), 3.

Marra M.C., Pardey P.G. et Alston J.M., 2003, «The payoffs to transgenic field crops: an assessment of evidence », AgBioForum 5, 2, pp. 43-50.

Mazoyer M., 2000, «La moitié de la paysannerie mondiale n'est pas solvable pour les grands laboratoires », Le Monde, édition électronique du 16/10/ 2006.

Moorjani N., 2005, «U.S. Announces Launch of West Africa Cotton Improvement Program: Another Step By the U.S. to Address Concerns of Key Cotton Countries », http://www.usda.gov/wps/ portal/usdahome?contendidonly $=$ true\&contentid=2005/11/0486.xml

Morse S., BennetT R. et ISMaËL Y., 2004, "Why Bt cotton pays for small- scale producers in South Africa ", $e$ Biotechnology 22, 4, pp. 379-380.

MYers D., 1999, "GM cotton fails to impress ", Pesticides News, 44, p. 6.

ORPHAL J., 2005, « Comparative analysis of the economics of $\mathrm{Bt}$ and Non-Bt cotton production », Institute of economics in Horticulture, Univ. of Hannover, Hannover, Germany, Pesticide Policy Project Publication Series. Special Issue, $\mathrm{N}^{\circ}$ 8, January 2005, p. 71.

Pray C.E., huang J., Hu R. et RozelLE S., 2002, «Five years of Bt cotton in China - the benefits continue ", The Plant Journal 31, 4, pp. 423-430.

PsChORN-STRauss E., 2004, « Bt cotton and small-scale farmers in Makhatini - a story of debt, dependency, and dicey economics », http:www.grain.org/ research/btcotton.cfm?id $=100$.

SAMPhier T. et CARLILE L., 2006, « African farmers say GM crops are not the way forward», http://www.iied.org/ mediaroom/release/29106.html.

QAim M. et DE JANVRY A., 2003, "Genetically modified crops, corporate pricing strategies, and Farmers' adoption: the case of Bt cotton in Argentina ", Amer. J. Agr. Econ. 85, 4, pp. 814828.

The Mitchell Group INC., 2004, Résumé et résultats de l'évaluation du secteur cotonnier en Afrique de l'Ouest, p. 36.

Traxier G. et Godoy-Avila S., 2004, "Transgenic cotton in Mexico », AgBioForum 7, 1-2, pp. 57-62.

Xu J., You Z., Wang W. et Yang Y., 2004, «Economic analysis of Bt cotton Planting in Jiangsu ", Journal of Yangzhou University (Agricultural and Life Science Edition) 25, 3, pp. 65-69. 
